\title{
Forms of mental phenomena
}

Artificial Intelligence and Natural Man. By Margaret Boden. Pp. 533. (Harvester Press: Sussex, 1977.) Hardback $£ 13.50$; paperback $£ 4.95$.

ARISTOTLE, I believe, characterised form as being "all of the boat that is not the wood". Artificial intelligence may illuminatingly be looked upon as the study of all of thinking that is not physical process. To understand and make use of form, we have to resort to representations of it-for instance, in the case of the boat, to a sketch, a blue-print or a verbal description. The best and most pregnant kind of representation man has yet found for exhibiting the forms of thought is a computer program. This is due firstly to the apparently limitless precision and power of programming languages for the description of processes of any degree of complexity and at any level of detail. But, more importantly, it is due to the fact that such a representation, though not that of a physical process, can be imposed upon and direct the physical processes of a computer, generating intelligible behaviour. Thereby, the criterion of science can be applied to such a representation: does it work? One cannot sail in the blueprint of a boat, but one can, for example, converse on some subject matter with a successful natural language understanding program, or one may have a program-on a computer provided with suitable sensor and motor peripherals-carry out in a versatile way many assembly tasks.

The main content of Dr Boden's book is a careful account and examination of a selection of representative pieces of work that have been done in trying to tease out the forms of mental phenomena like the understanding of natural language, visual perception, belief systems, neurosis, learning, problem solving and creativity. It is one of a rapidly increasing number of introductory accounts of the subject being published, the two best until now probably being American: Winston's Artificial Intelligence and Raphael's The Thinking Computer. These and Dr Boden's are genuinely introductory, in that they assume in the reader not only no knowledge of their subject but even no or hardly any knowledge of computers and how they work; the three differ in the amount of the latter included.
What mainly distinguishes the present book, however, is that it presents the view of an outsider, rather than a practitioner, of the discipline. This is responsible for its strengths, and for weaknesses to a leseer degree. The author's background of academic philosophy and psychology, as well as her humanist outlook, not only helps her not to let techniques rather than ideas dominate the presentation, but also is presumably responsible for its careful, critical and comprehensive scholarship, of a kind very rare in a brash, new, vigorous subject of this kind. The claims that are and might be made on behalf of any of the many cognitive models she describes are usually cut down to size, and of ten made the more convincing and impressive by that; and as for reading, the 34 pages of notes on the text in small print at the end of the book are a mine of information to guide one in the literature of and around the subject.

The writing is very clear and often elegant, though occasionally pedagogical to the point of coyness; but though the contents of the pieces of work she describes are so well presented, little of the excitement that may accompany the exploration of new intellectual territory is conveyed. The organisation of the expository part of the book is what is called in the jargon of the subject "top-down"; thus, the account starts with Colby's simulation of neurosis, reaches vision programs about half-way through, and reserves some account of modern "non-deterministic" programming languages only to the very end. There is some lack of historical perspective: for example, I think neither Leibniz nor Babbage is even mentioned.

But the book has a secondary purpose besides exposition. To quote from the preface: "I have tried to convey a sense of the relevance of artificial in- telligence to the understanding of natural man. Contrary to what most people assume, this field of research has a potential for counteracting the dehumanising influence of natural science. . . The common view that machine research must tend to display us humiliatingly to ourselves as "mere clockwork" is false. The more widely this is realised, the less of a threat will artificial intelligence present to humane conceptions of society." Although one may strongly doubt that "dehumanisation" in our society is due to the influence of natural science, Dr Boden does argue her general point well. This is done both implicitly in the main expository part of the text (accountingapart from its pedagogical advantageperhaps for the top-down approach referred to above), and in a final section confronting directly psychological, philosophical and social questions raised. This section ranges widely and interestingly, for example, over the mind-body "problem", possible limitations on the computational representatation of thought, aspects of the modelling of emotion. possible beneficial applications in education, and other applications elsewhere.

Boden is probably too conservative in her assessment of the likely effect on our culture of the development of artificial intelligence. And she incidentally answers in some detail many of the criticisms voiced by Dreyfus and others hostile to the subject. For example, she shows how tasks confidently claimed by some of the critics to be beyond the power of computational representation, like the understanding of metaphor or the use of what Polanyi called "tacit" knowkedge, are being broached in existing programs.

Bernard Meltzer is Professor of Computational Logic at the University of Edinburgh, UK.

\section{Handbook of human evolution}

Guide to Fossil Man. (Third edition.) By Michael H. Day. Pp. xviii +346 . (University of Chicago Press: Chicago; Cassell: London, 1977.) $\$ 15 ; £ 7.95$.

THE fossil evidence for human evolution has been accumulating for almost a century and a half, and throughout most of this period the remains of fossil man have appeared in a slow, if not leisurely, procession. Thus, the slowly emerging record of human evolution could easily be assimilated by specialist, student and layman alike. However, beginning about 1960 the study of fossil man entered a period of remarkable and dynamic productivity. Within the past 18 years more than 500 new hominid fossils have been 